УДК $616.314-089.28-036.83$

DOI 10.18413/2687-0940-2020-43-2-257-264

\title{
РЕАБИЛИТАЦИЯ ПАЦИЕНТОВ С ХРОНИЧЕСКИМИ ЗАБОЛЕВАНИЯМИ СЛИЗИСТОЙ ОБОЛОЧКИ ПОЛОСТИ РТА НА ФОНЕ СНИЖЕННОГО ИММУНИТЕТА В ПРАКТИКЕ ВРАЧА СТОМАТОЛОГА-ОРТОПЕДА
}

\section{REHABILITATION OF PATIENTS WITH CHRONIC DISEASES OF THE ORAL MUCOSA AGAINST THE BACKGROUND OF REDUCED IMMUNITY IN THE PRACTICE OF A DENTIST-ORTHOPEDIST}

\author{
Ю.Н. Котенева ${ }^{1,2}$, С.Н. Гонтарев ${ }^{1,2}$, А.А. Богданова ${ }^{1,2}$, В.В. Кострыкин ${ }^{1}$, \\ C.В. Макова ${ }^{1}$ \\ Yu.N. Koteneva ${ }^{1,2}$, S.N. Gontarev ${ }^{1,2}$, A.A. Bogdanova ${ }^{1,2}$, V.V. Kostrykin ${ }^{1}$, S.V. Makova ${ }^{1}$ \\ ${ }^{1}$ ООО ССБ «Стоматологическая поликлиника Старооскольского городского округа», \\ Россия, 309516, г. Старый Оскол, мкр. Ольминского, д. 6а \\ ${ }^{2}$ Белгородский государственный национальный исследовательский университет, \\ Россия, 308015, г. Белгород, ул. Победы, д. 85 \\ ${ }^{1}$ Social dentistry of Belogorye «Dental clinic of Stary Oskol urban district», \\ 6a Olminsky microdistrict, Stary Oskol, 308015, Russia \\ ${ }^{2}$ Belgorod National Research University, \\ 85 Pobedy St., Belgorod, 308015, Russia \\ E-mail: koteneva.iulya@yandex.ru
}

\begin{abstract}
Аннотация
В данной статье представлены результаты исследований, проведенных в 2018-2019 гг. на базе ООО «ССБ Стоматологической поликлиники Старооскольского района». Разработаны и описаны способы быстрой адаптации пациентов после протезирования съемными конструкциями на фоне сниженного иммунитета. Профилактика и лечение основывается при комплексном подходе к лечению с применением мягкого базисного материала «Ufigel» и иммуномодулирующего препарата «Имудон». В исследовании принимали участие 40 пациентов (20 мужчин и 20 женщин) в возрасте от 45 до 70 лет с вторичной частичной или полной адентией. К первой группе отнесли пациентов условно здоровых, которым впервые было проведено ортопедическое лечение съемными протезами. У пациентов 2-й группы в ходе сбора анамнеза были выявлены сопутствующие соматические заболевания, хронические стоматиты. Для данных больных использовали мягкую прокладку «Ufigel». К 3-й группе относились пациенты с хроническими заболеваниями полости рта, которые в адаптационный период 30 дней по назначению принимали курс иммуномодулирующего препарата «Имудон», а также использовали мягкую прокладку. Оценивали реакцию слизистой оболочки протезного ложа (в 3,7, 27 сутки). Приведенные в статье исследования должны повысить уровень ортопедического лечения съемными конструкциями. Изучено влияние иммуномодулятора «Имудон» и мягкого прокладочного материала «Ufigel» на адаптационный период пациентов со съемными конструкциями на фоне сниженного иммунитета.
\end{abstract}

\begin{abstract}
This article presents the results of research conducted in 2018-2019 on the basis of LLC «SSB of the Dental clinic of Starooskolsky district». Methods of rapid adaptation of patients after prosthetics with removable structures against the background of reduced immunity have been developed and described. Prevention and treatment is based on a comprehensive approach to treatment, using a soft base material «Ufigel» and immunomodulating drug «Imudon». The study involved 40 patients $(20$ men and 20 women) aged 45 to 70 years with secondary partial or complete adentia. The first group included patients who were conditionally healthy and had their first orthopedic treatment with removable prostheses. In group 2 patients, concomitant somatic diseases and chronic stomatitis were detected during
\end{abstract}


the collection of anamnesis. For these patients, a soft UFI gel pad was used. Group 3 included patients with chronic diseases of the oral cavity, which, in the adaptation period of 30 days, took an immunomodulating drug as prescribed «Imudon», and also used a soft pad. The reaction of the prosthetic bed mucosa was evaluated (on the 3, 7 and 27 days). The research presented in the article should increase the level of orthopedic treatment with removable structures. The influence of the immunomodulator «Imudon» and soft padding material «Ufigel» on the adaptation period in patients with removable structures, against the background of reduced immunity, was studied.

Ключевые слова: протезирование, сниженный иммунитет, иммуномодулирующая терапия, съёмные конструкции, заболевания слизистой оболочки полости рта.

Keywords: prosthetics, reduced immunity, immunomodulatory therapy, removable structures, diseases of the oral mucosa.

\section{Введение}

В отделение ортопедической стоматологии зачастую обращаются пациенты с частичным или полным отсутствием зубов, у 5-6 \% данных пациентов при проведении диагностических мероприятий выявляются хронические патологии слизистой оболочки полости рта, зачастую клиническая картина осложняется сопутствующими заболеваниями разных систем организма, что напрямую влияет на иммуностатус пациента [Бавыкина, Ефремова, 2011, с. 236-238]. Врачу стоматологу-ортопеду при протезировании съёмными конструкциями тяжело прогнозировать благоприятный исход лечения, так как приходится сталкиваться со многими неблагоприятными факторами: повышенный рвотный рефлекс, психологические расстройства, первый опыт использования съёмных протезов [Берсанов, 2015]. Следует не забывать, что высокий уровень защиты полости рта определяет иммунная система, которая обозначает резистентность организма к любому чужеродному материалу и играет важную роль в развитии хронических заболеваний слизистой оболочки полости рта.

К системе местной защиты полости рта относим: эпителий слизистой оболочки, слюну. Слюна - биологическая жидкость, от ее качества зависит сила местного иммунитета в полости рта, в которой определяется большое количество активных компонентов, обладающих своим особым защитным действием. Имуноглобулин sIgA препятствует адгезии микроорганизмов, лизоцим оказывает разрушительное действие на стенки бактерий, антимикробный белок лактоферрин обладает бактериостатическими свойствами, является маркером воспаления. Антимикробные пептиды, секретируемые эпителием полости рта, начинают иммунный ответ, а также регулируют воспалительный процесс. Качество и количество слюны претерпевает изменения с возрастом, от этого биологические вещества слюны варьируют в разных значениях: у пожилых людей в ротовую полость из кровотока синтезируется меньше белка и а-амилазы, тем самым снижая резистентность слизистой в адаптационный период пользования съемными конструкциями [Рыжова, Гонтарев, 2017].

Внедрение в практику иммуномодулирующего препарата «Имудон» в качестве лекарственного вещества воздействует на организм в целом. Вопросы профилактики и лечения воспалительных заболеваний слизистой полости рта являются актуальными для исследования, направленного на совершенствование оказания ортопедической, стоматологической помощи пациентам с хроническими заболеваниями слизистой оболочки полости рта, сокращая сроки адаптации.

Цель работы - определить потребность лечебно-профилактических мероприятий, направленных на улучшение адаптации к съемным конструкциям у пациентов с хроническими заболеваниями слизистой оболочки полости рта на фоне сниженного иммунитета. 


\section{Материалы и методы исследования}

Для нашего исследования было выбрано 40 пациентов, диагностические мероприятия, направленные на изучение слизистой, проводили до протезирования и в отсроченные периоды на 3, 7 и 21 сутки после наложения протезов на слизистую оболочку протезного ложа. Лечение начиналось с диагностических мероприятий, мы проводили субъективные и объективные обследования. К субъективным методам относятся сбор анамнеза жизни пациента, анамнез данного заболевания, а также жалобы больного [Жолудев, Гетте, 2016].

К объективным методам относим внешний вид больного и оцениваем слизистую оболочку по Суппле, особое внимание уделяем прикреплению щечных тяжей и складок, наличие или отсутствие воспалительных изменений на слизистой, внешний вид языка (выраженность сосочков, цвет и размер) [Gabardo et al., 2015].

Чтобы оценить показатели клеточных факторов местного иммунитета, использовали биологический материал пациента - кровь и слюну. Определение количественного содержания сывороточных иммуноглобулинов $\operatorname{IgA}, \operatorname{IgG}, \operatorname{IgM}, \operatorname{IgE}$ проводили методом радиальной иммунодиффузии по Manchini. Метод основан на количественном определении антиген - антитело [Семенюк и др., 2017]. При добавлении иммунной сыворотки в расплавленный агаровый гель, после затвердения происходило образование комплекса $\mathrm{A} Г+\mathrm{AT}$, оседающего в виде преципитата, диаметр которого прямо пропорционален количеству соответствующего иммуноглобулина в образце [Китаева и др., 2015]. Иммуноферментный анализ смыва из ротовой полости на определение лизоцимной активности осуществлялся путем сбора слюны через 3 часа после приема пищи и предварительного полоскания рта физиологическим раствором. Перед данной процедурой исключались все провоцирующие факторы, препятствующие секреции слюнных желёз. Полученную жидкость собирали в стерильную пробирку, определяя процентное соотношение IgA, sIgA. Исследование фагоцитарной активности лейкоцитов - лабораторный показатель, который отражает функциональные способности нейтрофилов и моноцитов [Гонтарев и др., 2016].

\section{Результаты исследования и их обсуждение}

Пациенты были разделены на 3 группы:

1 группа - пациенты, впервые протезированные съемными конструкциями, в период адаптации были проведены традиционные методы коррекции (17 чел.);

2 группа - к данной группе отнесли пациентов с хроническими стоматитами в полости рта, в период адаптации применялась мягкая прокладка;

3 группа - пациенты, которым после наложения протезов назначили иммуномодулирующий препарат «Имудон», а также использовалась мягкая прокладка (10 чел).

После протезирования на слизистой оболочки протезного ложа выявляются патологические элементы: без видимых изменений эпителиального слоя слизистой, а также с нарушением целостности эпителия и выраженным стоматитом [Гонтарев и др., 2018].

Следует отметить, что у всех пациентов перед протезированием прошло в достатке времени после удаления зубов, костные выступы (экзостозы) устранялись хирургическим путем. При объективной оценке слизистой оболочки учитывали 3 основных показателя (болевой синдром, увлажненность слизистой оболочки протезного ложа, наличие воспалительных изменений в эпителии), указанных в таблице [Itakura et al., 2018]. Пациенты после протезирования предъявляли сходные клинические жалобы: боли под базисом протеза в состоянии покоя и нарастающие во время еды, повышенное слюноотделение, повышенный рвотный рефлекс, а также расстройства психологического характера [Цимбалистов и др., 2011]. 
Характеристика клинических показателей слизистой оболочки полости рта

Characteristicclinical findings of mucous membrane of the oral cavity

\begin{tabular}{|c|c|}
\hline Клинический показатель & Значения показателя \\
\hline 1. Болевой синдром & $\begin{array}{l}0 \text { - отсутствуют; } \\
1 \text { - умеренно выраженный болевой синдром во время } \\
\text { пережевывания пищи; } \\
2 \text { - сильно выраженный болевой синдром при жевании; } \\
3 \text { - боли постоянные при ношении; } \\
4 \text { - выраженный болевой синдром при разговоре. }\end{array}$ \\
\hline $\begin{array}{l}\text { 2. Влажность слизистой } \\
\text { оболочки протезного ложа и } \\
\text { цвет }\end{array}$ & $\begin{array}{l}0 \text { - влажная, бледно-розовая; } \\
1 \text { - сухая потеря блеска, анемичная; } \\
2 \text { - умеренно влажная, гиперемированная. }\end{array}$ \\
\hline 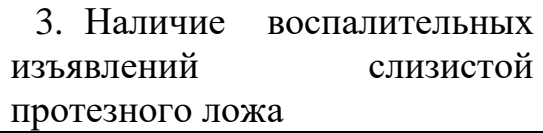 & $\begin{array}{l}0 \text { - без патологии; } \\
1 \text { - гипермия слизистой в отдельных участках, эрозии и афты: } \\
2 \text { - разлитая гиперемия слизистой с язвенными поражениями. }\end{array}$ \\
\hline
\end{tabular}

Условно единицы $(1,2,3)$ перевели в процентное соотношение. Оценка результатов проводилась на третьи сутки, основная часть $(60 \%-70 \%)$ пациентов относилась к 1-й и 2-й группе, и лишь 30 \% являлись пациентами 3-й группы. На 7-е сутки с жалобами на жжение, наличием язв и разлитой гиперемией на слизистой оболочке полости рта 50 \% обращений было из 1-й группы, и только $25 \%$ - из 2-й группы. К 21-м суткам в адаптационном периоде от начала пользования съемными конструкциями в первой группе (где проводились традиционные методы коррекции) реакция слизистой оболочки сохранялась у 60 \% исследуемых. При обследовании слизистой 3-й группы пациентов, получивших в адаптационный период лекарственную терапию препаратом «Имудон» и применения подкладочного материала «Ufigel», на 3-и сутки не выявили значительных повреждений эпителия протезного ложа, лишь наблюдалась точечная гипермия у 20 \% пациентов, что в 4 раза меньше по сравнению с 1-й группой и в 2,5 раза меньше, чем во 2-й группе [Пономарев, 2014].

Клинические наблюдения и объективные изучения слизистой оболочки протезного ложа в разных группах дают возможность проанализировать положительную динамику адаптации к съемным протезам [Гонтарев и др., 2016]. У пациентов с применением мягкого подкладочного материала «Ufigel» в сочетании с иммуномодулирующим препаратом «Имудон», который обладает выраженным кератопластическим и противовоспалительным действием, сводится к минимуму развитие хронических стоматитов в полости рта [Каливраджияна и др., 2020].

Таким образом, анализ проведенного лабораторного исследования показывает существенные различия в период адаптаций к съемным пластиночным протезам, из-за особенностей иммунологической реакции пациентов. В группе пациентов с хроническими стоматитами секретируемый $\operatorname{sg}$ А значительно снижен по сравнению с нормальными показателями во 2-й и 3-й группах обследуемых пациентов [Гонтарев и др., 2018]. На 7 сутки после протезирования у больных 2-й группы протезировавшихся впервые содержание сывороточных иммуноглобулинов IgM, sIgG превышено, нормализуется их содержание через месяц. Это является показателем при первичном воздействии базиса на слизистую полости рта, мягкая подкладка «Ufigel» служит для уменьшения давления на слизистую оболочку, тем самым предотвращая появление патологических изменений на протезном ложе [Inderdeep et al., 2017].

Пациенты 3-й группы после повторного ортопедического лечения, принимающие лекарственную иммуномодулирующую терапию, по всем показателям в пределах нормы. Концентрация лизоцима $\operatorname{IgA}, \operatorname{sgA}$ в слюне в данной группе обследуемых пациентов резко 
увеличена, что является показателем сильно выраженной воспалительной реакции в первые 2 недели пользования пластиночными съемными протезами. В 1-й обследуемой группе пациентов с хроническими стоматитами слизистой оболочки полости рта в смывах из полости рта наблюдалось IgE выше средней границы (120 ME/мл) по сравнению с уровнем данных показателей до протезирования, данные исследования показывают аллергическую реакцию слизистой. Также данные показали умеренно выраженное увеличение уровня лизоцима и стабильные показатели фагоцитоза. Спад фагоцитарной активности нейтрофилов говорит об угнетении местного иммунитета, к этому приводит травматическое воздействие базиса протеза на слизистую оболочку полости рта. Спустя месяц после протезирования показатели 2-й и 3-й группы нормализуются, что говорит об адаптации органов и слизистых оболочек полости рта к съемным конструкциям [Gates et al., 2014].

\section{Выводы}

Делаем выводы, что у пациентов с хроническими заболеваниями слизистой оболочки применение иммуномодулирующего препарата «Имудон» и подкладочного материала «Ufigel» на разных этапах пользования съемными пластиночными протезами способствует не только исчезновению симптомов в адаптационный период, но и значительно усиливает процессы регенерации.

Нарушение целостности эпителиального покрова под компрессией съемной конструкции диктует необходимость в применении данных препаратов, благоприятно влияя на сроки адаптации, нормализуя показатели местного иммунитета полости рта.

Препарат «Имудон» - бактериального происхождения, тем самым отличается своим составом от других иммуномодуляторов. В его состав входят бактерии, при попадании на слизистую в небольшом количестве активно стимулирующие выработку иммуноглобулина и лизоцима, которые являются основой естественного иммунитета. Для улучшения клинической картины для перебазировки используем мягкий подкладочный материал «Ufigel» на силиконовой основе, наши данные показывают снижение раздражения слизистой.

В исследуемых группах нами было выявлено повышение значений целого ряда показателей: ФИ, иммуноглобулинов IgA, G. Это свидетельствует об активации клеточного звена иммунитета полости рта, способствуя усилению функциональной (фагоцитарной) активности нейтрофильных гранулоцитов в полости рта. А также усиливается функциональная активность гуморального звена местного иммунитета, об этом говорит стойкое повышение s-IgA в ротовой жидкости.

\section{Заключение}

Проблема адаптации пациентов к съемным ортопедическим конструкциям является очень важной, так как напрямую влияет на благоприятный прогноз лечения. Планирование рационального протезирования начинаем с предварительной санации всех очагов воспаления в полости рта, симптоматическая терапия проводится по показаниям.

Ортопедическое лечение пациентов с хроническими заболеваниями слизистой оболочки полости рта может проводиться как в период ремиссии, так и в фазу обострения. Таким образом, применение препаратов «Имудон» и «Ufigel» устраняет провоцирующие факторы, пагубно влияющие на процесс привыкания к протезам.

Простота в применении данных препаратов и эффективность дает нам возможность рекомендовать в клиническую практику.

Помимо профилактических и лечебных мер, применяющихся в период адаптации, немаловажная роль отводится индивидуальному психоэмоциональному статусу пациента.

Главная задача врача стоматолога-ортопеда - правильно мотивировать пациента и помочь осознать необходимость лечения, вне зависимости от того, с какими сложностями придется столкнуться при приспособлении к съемной конструкции. Протокол ведения ор- 
топедически больного пациента, к сожалению, не включает на этапе диагностики определение психологических особенностей пациента.

\section{Список литературы}

1. Бавыкина Т.Ю., Ефремова О.А. 2011. Полость рта - зеркало заболеваний внутренних органов. Научные ведомости Белгородского государственного университета. Серия: Медицина. Фармация. 10 (105): 236-238.

2. Берсанов Р.У. 2015. Влияние современных конструкций протезов на динамику показателей качества жизни пациентов. Российский стоматологический журнал, 6: 43-44.

3. Гонтарев С.Н., Гонтарева И.С., Замулин Д.О., Никишаева А.В., Федорова И.Е. 2016. Патология слизистой оболочки полости рта токсико-аллергического генеза при ортопедическом лечении. Актуальные вопросы клинической стоматологии, сборник научных работ, с. 43-47.

4. Гонтарев С.Н., Гонтарева И.С., Никишаева А.В. 2016. Использование фитопрепаратов в стоматологии детского возраста. Научный результат, серия Медицина и фармация, 2 (2): 17-21.

5. Гонтарев С.Н., Гонтарева И.С., Чуев В.П., Никишаева А.В., Банчук А.Ю., Моисеев И.О. 2016. Анализ сравнительной клинической эффективности герметизации фиссур при использовании препаратов фото и химической полимеризации. Научный результат, серия Медицина и фармация, 2 (2): 22-26.

6. Гонтарев С.Н., Хлебникова И.Г., Моисеева М.А., Моисеев И.О., Котенёва Ю.Н., Гонтарева И.С., Ефимова А.С. 2018. Использование методики избирательного пришлифовывания при заболеваниях парадонта как один из основных методов лечения. Стоматология славянских государств, с. $70-71$.

7. Жолудев С.Е., Гетте С.А. 2016. Решение проблемы адаптации к съемным конструкциям зубных протезов при полной утрате зубов (клинический случай). Проблемы стоматологии, 3(12): 46-51.

8. Каливраджияна Э.С., Лебеденко И.Ю., Брагина Е.А., Рыжовой И.П. 2020. Ортопедическая стоматология. ГЭОТАР-Медиа: 800 с.

9. Китаева Т.А., Данилина Т.Ф., Голубев А.Н. 2015. Современные аспекты лечения геронто-стоматологических пациентов съемными пластиночными протезами. Dental Tribune Russia.

10. Пономарев С.А. 2014. Осложнения, клинические и технологические ошибки при ортопедическом лечении больных съемными зубными протезами и их профилактика: дис. ... канд. мед. наук. Омск: 119 с.

11. Рыжова И.П., Гонтарев С.Н., Новожилова М.С., Погосян Н.М. 2017. Влияние ортопедических конструкций на микробиоценоз полости рта. Современная ортопедическая стоматология, 28: 24-26.

12. Семенюк В.М., Ахметов Е.М., Федоров В.Е., Качура Г.П., Ахметов С.Е. 2017. Результаты организации, эффективности ортопедического лечения и качества зубных протезов (данные социологического исследования). Институт Стоматологии, 1: 26-29.

13. Цимбалистов А.В., Жданюк И.В., Иорданишвили А.К. 2011. Роль подготовительных мероприятий в возникновении дефектов протезирования съемными зубными протезами. Институт Стоматологии, 1: 49-50.

14. Inderdeep S.W., Lovleen B., Amritpal S., Kamaldeep K., Aditya D. 2017. Prevalence of Medical Comorbidities in Dental Patients. Ann. Int. Med. Den. Res., 3 (1).

15. Itakura S., Miyata M., Kuroda A., Setoguchi M., Kusumoto A., Hokonohara D., Ohishi M. 2018. The association of Bite Instability and Comorbidities in Elderly People. Intern. Med. 57 (11): $1569-1576$.

16. Gabardo M.C., Moyses S.J., Moyses S.T., Olandoski M., Olinto M.T., Pattussi M.P. 2015. Multilevel analysis of self-perception in oral health and associated factors in Southern Brazilian adults: a cross-sectional study. Cad. Saude. Publica. 31 (1): 49-59.

17. Gates W.D., Cooper L.F., Sanders A.E., Reside G.J., De Kok I.J. 2014. The effect of implant-supported removable partial dentures on oral health quality of life. Clin. Oral. Implants. Res. 25 (2): 207-213. 


\section{References}

1. Bavykina T.Ju., Efremova O.A. 2011. Polost' rta - zerkalo zabolevanij vnutrennih organov [Oral cavity - a mirror of internal diseases]. Nauchnye vedomosti Belgorodskogo gosudarstvennogo universiteta. Serija: Medicina. Farmacija. 10 (105): 236-238.

2. Bersanov R.U. 2015. Vlijanie sovremennyh konstrukcij protezov na dinamiku pokazatel kachestva zhizn ipacientov [Influence of modern prosthetic designs on the dynamics of the quality of life of patients]. Rossijskij stomatologicheskij zhurnal, 6: 43-44.

3. Gontarev S.N., Gontareva I.S., Zamulin D.O., Nikishaeva A.V., Fedorova I.E. 2016. Patologija slizistoj obolochki polosti rta toksiko-allergicheskogo geneza pri ortopedicheskom lechenii [Pathology of the oral mucosa of toxic-allergic origin in orthopedic treatment]. Aktual'nye voprosy klinicheskoj stomatologii, sbornik nauchnyh rabot: c. 43-47.

4. Gontarev S.N., Gontareva I.S., Nikishaeva A.V. 2016. Ispol'zovanie fitopreparatov v stomatologii detskogo vozrasta [The use of herbal medicine in stomatology of children's age]. Nauchnyj rezul'tat, serija Medicina I farmacija, 2 (2): 17-21.

5. Gontarev S.N., Gontareva I.S., Chuev V.P., Nikishaeva A.V., Banchuk A.Ju., Moiseev I.O. 2016. Analiz sravnitel'noj klinicheskojj effektivnosti germetizacii fissur pri ispol'zovanii preparatov foto i himicheskoj polimerizacii [Analysis of comparative clinical effectiveness of fissure sealing when using photo and chemical polymerization preparations]. Nauchnyj rezul'tat, serija Medicina I farmacija, 2 (2): $22-26$.

6. Gontarev S.N., Hlebnikova I.G., Moiseeva M.A., Moiseev I.O., Kotenjova Ju.N., Gontareva I.S., Efimova A.S. 2018. Ispol'zovanie metodiki izbiratel'nogo prishlifovyvanija pri zabolevanijah paradonta kak odin iz osnovnyh metodov lechenija [The use of selective sanding techniques for periodontal diseases as one of the main methods of treatment]. Stomatologija slavjanskih gosudarstv, c. 70-71.

7. Zholudev S.E., Gette S.A. 2016. Reshenie problem adaptacii k s"emnym konstrukcijam zubnyh protezov pri polnoj utrate zubov (klinicheskij sluchaj) [ Solving the problem of adaptation to removable denture structures in case of complete loss of teeth]. Problemy stomatologii, 3 (12): 46-51.

8. Kalivradzhijana Je.S., Lebedenko I.Ju., Bragina E.A., Ryzhovoj I.P. 2020. Ortopedicheskaja stomatologija [Prosthetic dentistry]. GJeOTAR-Media: $800 \mathrm{~s}$.

9. Kitaeva T.A., Danilina T.F., Golubev A.N. 2015. Sovremennye aspekty lechenija gerontostomatolgicheskih pacientov s"emnymi plastinochnymi protezami [Modern aspects of treatment of gerontostomatological patients with removable plate prostheses]. Dental Tribune Russia.

10. Ponomarev S.A. 2014. Oslozhnenija, klinicheskie i tehnologicheskie oshibki pri ortopedicheskom lechenii bol'nyh s"emnymi zubnymi protezami i ih profilaktika [Complications, clinical and technological errors in orthopedic treatment of patients with removable dentures and their prevention]: dis. ... kand. med. nauk. Omsk: $119 \mathrm{c}$.

11. Ryzhova I.P., Gontarev S.N., Novozhilova M.S., Pogosjan N.M. 2017. Vlijanie ortopedicheskih konstrukcij na mikrobiocenoz polosti rta [The influence of orthopedic structures on the microbiocenosis of the oral cavity]. Sovremennaja ortopedicheskaja stomatologija, 28: 24-26.

12. Semenjuk V.M., Ahmetov E.M., Fedorov V.E., Kachura G.P., Ahmetov S.E. 2017. Rezul'taty organizacii, jeffektivnosti ortopedicheskogo lechenija i kachestva zubnyh protezov (dannye sociologicheskogo issledovanija) [Results of organization, effectiveness of orthopedic treatment and quality of dental prostheses]. Institut Stomatologii, 1: 26-29.

13. Cimbalistov A.V., Zhdanjuk I.V., Iordanishvili A.K. 2011. Rol' podgotovitel'nyh meroprijatij $\mathrm{v}$ vozniknovenii defektov protezirovanija semnymi zubnymi protezami [The role of preparatory measures in the occurrence of defects in prosthetics with removable dentures]. Institut Stomatologii, 1: 49-50.

14. Inderdeep S.W. Lovleen B., Amritpal S., Kamaldeep K., Aditya D. 2017. Prevalence of Medical Comorbidities in Dental Patients. Ann. Int. Med. Den. Res., 3 (1).

15. Itakura S., Miyata M., Kuroda A., Setoguchi M., Kusumoto A., Hokonohara D., Ohishi M. 2018. The association of Bite Instability and Comorbidities in Elderly People. Intern. Med. 57 (11): $1569-1576$.

16. Gabardo M.C., Moyses S.J., Moyses S.T., Olandoski M., Olinto M.T., Pattussi M.P. 2015. Multilevel analysis of self-perception in oral health and associated factors in Southern Brazilian adults: a cross-sectional study. Cad. Saude. Publica. 31 (1): 49-59.

17. Gates W.D., Cooper L.F., Sanders A.E., Reside G.J., De Kok I.J. 2014. The effect of implant-supported removable partial dentures on oral health quality of life. Clin. Oral. Implants. Res. 25 (2): 207-213. 


\section{Ссылка для цитирования статьи \\ Link for article citation}

Котенева Ю.Н., Гонтарев С.Н., Богданова А.А., Кострыкин В.В., Макова С.В. 2020. Реабилитация пациентов с хроническими заболеваниями слизистой оболочки полости рта на фоне сниженного иммунитета в практике врача стоматолога-ортопеда. Актуальные проблемы медицины, 43 (2): 257-264. DOI 10.18413/2687-0940-2020-43-2-257-264.

Koteneva Yu.N., Gontarev S.N., Bogdanova A.A., Kostrykin V.V., Makova S.V. 2020. Rehabilitation of patients with chronic diseases of the oral mucosa against the background of reduced immunity in the practice of a dentist-orthopedist. Challenges in Modern Medicine, 43 (2): 257-264 (in Russian). DOI 10.18413/2687-0940-2020-43-2-257-264. 